\title{
Correction: TRIM27 contributes to glomerular endothelial cell injury in lupus nephritis by mediating the Fox01 signaling pathway
}

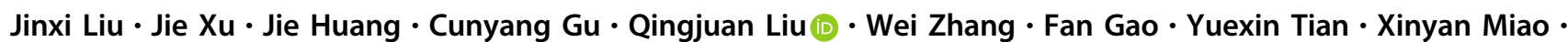
Zixuan Zhu $\cdot$ Baiyun Jia $\cdot$ Yu Tian · Lunbi Wu $\cdot$ Hang Zhao $\cdot$ Xiaojuan Feng $(1) \cdot$ Shuxia Liu $(\mathbb{D}$

Published online: 3 May 2021

(c) The Author(s), under exclusive licence to United States and Canadian Academy of Pathology 2021

Correction to: Laboratory Investigation

https://doi.org/10.1038/s41374-021-00591-9

The original version of this article unfortunately contained a mistake. Due to a typeset error the Summary was omitted. We apologize for the error. The original article has been corrected.

\begin{abstract}
Summary
The expression of TRIM27 in MRL/lpr mice and human renal glomerular endothelial cells cultured in vitro is significantly enhanced. Down-regulating the expression of TRIM27 inhibits the loss of glycocalyx and damage to endothelial cells through the FoxO1 pathway. In addition, inhibition of the protein kinase B (Akt) pathway can reduce the damage by mediating the expression of TRIM27.
\end{abstract}

